\title{
Survey to identify Mycobacterium leprae-infected household contacts of patients from prevalent regions of leprosy in Colombia
}

\author{
N Cardona-Castro/ ${ }^{+}$, JC Beltrán-Alzate', R Manrique-Hernández ${ }^{1}$ \\ Instituto Colombiano de Medicina Tropical, Universidad CES, Cra 43A No. 52Sur-99, Sabaneta, Antioquia, Colombia ${ }^{1}$ Universidad CES, \\ Medellín, Colombia
}

Leprosy in Colombia is in the post-elimination phase; nevertheless, there are regions of this country where the incidence is still around 3-4/100,000. Early detection of leprosy patients is a priority for achieving control and elimination of leprosy; however, the clinical exam is not very sensitive and thus, the majority of patients are diagnosed only when they demonstrate lesions, and damage to the nerves and skin has already occurred. The goal of the present study was to identify Mycobacterium leprae infection and immune responses in household contacts (HHC) of leprosy patients from three prevalent regions of Colombia. Clinical examination, the Mitsuda test, evaluation of IgM anti-PGL-I in the serum, the bacillar index (BI), and polymerase chain reaction (PCR) from nasal swabs (NS) were performed for 402 HHC of 104 leprosy patients during a cross-sectional survey. Positive titers for IgM anti-PGL1 were found for $54 \mathrm{HHC}$, and PCR-positive NS for 22. The Mitsuda reaction was negative for $38 \mathrm{HHC}$, although three were positive for IgM anti-PGL-1 titers. The data document that leprosy transmission among HHC is still occurring in a non-endemic country.

Key words: leprosy - Mycobacterium leprae - household contacts - IgM anti PGL-1 - Mitsuda reaction

Leprosy continues to be a public health problem in some countries (Gupta et al. 2004, Meima et al. 2004). The prevalence of leprosy in Colombia has decreased over the last 15 years. In 1985, the prevalence of leprosy was 5.5/10,000 and, during the last five years, it was less than 0.5/10,000 (http://www.minsalud.gov.co). The introduction of multiple-drug therapy (MDT) in Colombia in 1985 has resulted in a decrease in disease burden. Currently, according to the World Health Organization (WHO), leprosy in Colombia is not a public health problem, and control of leprosy is in the "postelimination phase" (http://www.who.org). However, the overall reduction in prevalence is not a real reflection of the problem with respect to population distribution. Colombia has some regions where the actual prevalence is higher than 1/10,000. Moreover, the "incidence" (new cases) of leprosy in some Colombian states was as high as 4.1/100,000 in 2004 (http://www.minsalud.gov.co).

Early detection of leprosy patients is a priority for achieving control and elimination of leprosy (Meima et al. 2004). Determination of the risk of an infected contact to develop leprosy is still unpredictable, and leprosy elimination is not possible as long as infected

Financial support: Gorgas Memorial Institute Research Award of the American Society of Tropical Medicine and Hygiene 2002, Dirección Seccional de Salud de Antioquia, Instituto Colombiano de Medicina Tropical - Universidad CES.

+ Corresponding author: ncardona@ces.edu.co

Received: 21 November 2007

Accepted: 9 June 2008 contacts could develop the disease in the future (Lockwood 2002). The compulsory plan for health attention in Colombia includes the clinical examination of household contacts (HHC) at the time of index case diagnosis (Kalk \& Fleisher 2004). While this is a sound concept for surveillance, leprosy has a long latency period, and several years of follow-up is necessary to detect early stages of disease (Lockwood \& Kumar 2004).

In a similar study designed to detect Mycobacterium leprae infection in $\mathrm{HHC}$ in a non-prevalent region of Colombia, we found two HHC with multibacillary leprosy (Cardona-Castro et al. 2005). These findings suggest that early diagnosis of leprosy in HHC through clinical examination at the time of index case diagnosis is not sufficient and that continued follow-up and testing using other available diagnostic tools is necessary. Diagnosis of leprosy is difficult; those exhibiting symptoms (tingling feeling in fingers, loss of sensation in extremities, skin patches, etc) may not ascribe them to leprosy, or patients may be misdiagnosed by physicians. Thus, diagnosis is often delayed, preventing the identification of risk factors and allowing the continued spread of disease (Lockwood 2002).

Pessimistic opinions about the "final push" proposed by WHO to eliminate leprosy are based not only on the high prevalence rates in some countries, but also on the scarce information about reservoirs and transmission, antibiotic resistance, and host factors governing immunity, especially in high risk populations, as those in contact with leprosy patients (Naafs 2000, Visschedijk et al. 2000, Gupta et al. 2004).

Currently, leprosy control programs in Colombia may not be using the most sensitive and up-to-date methods for early detection of leprosy. In addition to clinical examination, markers of infection such as polymerase 
chain reaction (PCR) and the immune response could greatly improve the early detection of HHC at risk for disease. The current study seeks to provide a systematic framework for the application of available tools to identify $M$. leprae infection and immune responses in household contacts of leprosy patients.

\section{SUBJECTS, MATERIALS AND METHODS}

A cross-sectional survey was carried out to detect infection and evaluate immune responses in $402 \mathrm{HHC}$ of 104 leprosy patients registered in the local leprosy control program. HHC were defined as individuals over four years of age without clinical signs or symptoms, but living in the same household as a patient with laboratory confirmed leprosy for more than two years. The study population was comprised of 13 towns located in three prevalent Colombian states: Bolívar (prevalence of 4/10,000), Córdoba and Sucre (2-3/10,000).

Ethical considerations - All participants were volunteers; they provided informed consent to participate and signed a document to this effect. Parents or guardians signed the informed consent for children under 18 years of age. The current study was approved by the Ethical Committee of Instituto Colombiano de Medicina Tropical and by the University CES.

ELISA test - All patients were assessed with the IgM anti-PGL-1 assay using the native PGL-1 antigen provided by Dr. Patrick Brennan from Colorado State University, USA, according to the methodology described previously (Cho et al. 1983). Briefly, $50 \mu \mathrm{l}$ of $2.0 \mu \mathrm{g} /$ $\mathrm{ml}$ PGL-I diluted 1:1000 was added to each well of a 96 -well Dynatech ${ }^{\circledR}$ plate. After incubation at room temperature, $100 \mu \mathrm{l}$ of $1 \%$ BSA-PBS was added and the plate was incubated at $37^{\circ} \mathrm{C} / 1 \mathrm{~h}$. Then, $50 \mu \mathrm{l}$ of serum sample diluted $1: 300$ in $0.5 \%$ BSA-PBS was added to the wells and incubated at $37^{\circ} \mathrm{C} / 1 \mathrm{~h}$. The wells were washed four times with PBS, incubated with $50 \mu \mathrm{l}$ of conjugated antihuman IgM antibody at $37^{\circ} \mathrm{C} / 1 \mathrm{~h}$, and washed four times with PBS. Finally, $50 \mu \mathrm{l}$ of TMB substrate was added, followed by incubation at $37^{\circ} \mathrm{C} / 10-15 \mathrm{~min}$. The reaction was stopped by adding $50 \mu \mathrm{l}$ of $2.0 \mathrm{M} \mathrm{H}_{2} \mathrm{SO}_{4}$, and measured with a spectrophotometer using a $450 \mathrm{~nm}$ filter. Each sample was tested in duplicate. The cut-off used to interpret positive and negative IgM anti-PGL-1 titers was calculated using serum samples from 75 volunteers representing the general population without leprosy contact. The results of this ELISA test were interpreted according to a calculated cut-off value of 0.115 (mean plus 3 standard deviations). IgM anti PGL-1 titers over 0.115 were considered to be positive.

Acid fast stain - Patients and HHC were tested for the bacillar index (BI) using the Ziehl Neelsen stain. Briefly, slit skin smears (SSs) on a microscopic glass slide were obtained from the earlobes (2), margins of lesions (2), or elbows (2) by puncture with a sterile lancet. Nasal mucus swab (NS) (1) was also obtained using cotton swabs. The SSs were examined for the presence of acid fast bacilli (AFB) and a bacillary index (BI) was recorded (Isenberg 1992).
$P C R$ - NS samples were collected from $76 \mathrm{HHC}$ and stored in $70 \%$ ethanol. DNA extraction was then carried out (Scollard et al. 1998, Jadhav et al. 2001). PCR testing was performed on each sample in duplicate using the primers and methodology previously described (Scollard et al. 1998, Groathouse et al. 2004). The 12-5 primer set for M. leprae which is specific for a single copy locus has a product length of $289 \mathrm{bp}$ and the following sequences: 5'CTGGTCCACTTGCGGTACG3' (forward), 5'GGAGAAGGAGGCCGAATACA3' (reverse). The PCR reaction tube contained a $50 \mu 1$ reaction comprised of $5 \mu \mathrm{l}$ of DNA and $45 \mu \mathrm{l}$ of PCR master mix. M. leprae DNA controls were included. PCR amplification was performed using standard conditions (Scollard et al. 1998). Products were visualized by agarose gel electrophoresis in $2 \%$ NuSieve GTG/SeaKem GTG (1:1) agarose gels (BioWhitaker, Rockland, USA) in $1 \mathrm{X}$ TAE buffer (4M Tris-acetate, pH 8.0, 1 mM EDTA; Invitrogen). Amplification products were electrophoresed in $2 \%$ agarose gels and stained with ethidium bromide (Scollard et al. 1998).

Lepromin A skin test - Lepromin A provided by the Schieffelin Leprosy Research and Training Centre, Karigiri, Vellore, India was used to test leprosy patients and HHC. Lepromin A $(0.1 \mathrm{ml}$ of 37 millions of bacilli/ $\mathrm{ml}$ ) was injected intradermally into the lateral side of the right forearm. Induration was measured 21-28 days after application, representing the Mitsuda reaction. Reactions of $4 \mathrm{~mm}$ or more were considered to be positive (Mitsuda 1953, Feitosa et al. 1996).

Clinical examination - Physical examination was carried out for all HHC to detect signs and symptoms indicative of leprosy in the skin and peripheral nerves. Patients were also examined in order to record lesions. This clinical examination was performed by specially trained physicians or health personnel.

Statistical analysis - Statistical analyses were performed using the EPI info 6.04 statistical program. Comparison between qualitative variables was performed using the Chi-square test; quantitative variables were compared by the Student's $t$ test; $p<0.05$ was considered statistically significant.

\section{RESULTS}

Characteristics of Leprosy patients - A total of 104 leprosy patients classified according to the Ridley-Joplin system were included. They were distributed among 80 houses: 63 houses had only one leprosy patient, 14 had two, three had three, and two houses had four and six patients, respectively. The majority of the patients were male $(n=64)$, and ages ranged from 8-87 years, with a median of 49 years. Four children were identified with leprosy; three were boys aged eight, 11 , and 16 years, and one was a 12-year-old girl. All had tuberculoid leprosy diagnosis. Physical changes such as hypochromic patches and decreased sensation in the extremities were most common. Malformations and functional sequelae were found in 31 patients. 
Positive titers for IgM anti-PGL1 were detected in 33 (32\%) patients; $25 \mathrm{LL}, 7 \mathrm{BL}$, and $1 \mathrm{BB}$ patient $(\mathrm{p}<0.0001)$. A negative Mitsuda reaction was obtained for LL 25 patients, BL in 11 , and BB in 16 ( $p=0.00004)$. The Bacillar index, IgM anti-PGL-I antibodies, and Mitsuda test were conducted for the leprosy patients at the time of the survey. Table I shows the test results according to clinical classification.

Characteristics of the HHC - $402 \mathrm{HHC}$ were studied, providing a ratio of $3.8 \mathrm{HHC}$ per leprosy patient. The majority of $\mathrm{HHC}$ were women (277). HHC ages ranged from 4-95 years old, with a median of 22 years. Table II shows the characteristics of the HHC according to the $\mathrm{BI}$ and clinical diagnosis of the leprosy patients. A total of $126(31.4 \%)$ individuals were contacts of BI-positive patients; $50 \mathrm{HHC}$ had contact with two or more patients in the same household; $31 \mathrm{HHC}$ cohabitated with two leprosy patients, nine $\mathrm{HHC}$ with four, three $\mathrm{HHC}$ with three, and seven HHC with six. Positive titers for IgM anti-PGL-I were found in 54 (13.4\%) HHC; 22 were contacts of BI-positive patients and 32 were contacts of BInegative patients $(\mathrm{p}>0.05)$. Twenty-five $(46 \%)$ of the $54 \mathrm{HHC}$ with positive titers for IgM anti-PGL-I were children under 15 years of age, the youngest being five years old; 12 of these (48\%) were contacts of BI-positive patients, and $13(52 \%)$ were contacts of BI-negative pa- tients ( $p>0.05)$. Twenty-nive of 80 houses $(36,2 \%)$ had at least one anti-PGL1 IgM-positive HHC; 15 houses had one seropositive HHC, nine houses had two, two houses had three, and three houses had four, five and six seropositive $\mathrm{HHC}$ each. With respect to the Mitsuda reaction, $38 \mathrm{HHC}$ were Mitsuda reaction-negative, though three of these were seropositive for IgM anti-PGL1 titers.

Twenty-two of $71(31 \%)$ NS samples tested positive by PCR, and 16 of these were anti-PGL1 IgM-positive.

Eighteen HHC showed skin signs indicative of leprosy. Their clinical diagnoses included cutaneous mycosis, vitiligo, and dermatitis. Neural signs of leprosy were not found. No HHC was diagnosed as leprosy patient.

\section{DISCUSSION}

Leprosy control strategies seek to stop transmission through early case detection and treatment with MDT. However, the number of new cases has not declined in several years, indicating that transmission continues (Meima et al. 2004). The available diagnostic tests used to detect infection lack the sensitivity and specificity necessary for early diagnosis of leprosy. Our study made use of several tests to improve the diagnostic accuracy of leprosy infection in HHC of leprosy patients.

Our study shows that the IgM-positive titers in HHC were not associated with the $\mathrm{BI}$ or clinical diagnosis of the patient. This suggests that contact with M. leprae can

TABLE I

Characteristics of leprosy patients according to test results

\begin{tabular}{lcccc}
\hline & \multicolumn{3}{c}{ Patients characteristics } \\
\cline { 2 - 5 } Patient diagnostic & $\mathrm{n}(\%)$ & BI positive (n) & IgM anti PGL1 titers positive (n) & Mitsuda negative (n) \\
\hline Ridley Joplin & $44(42.2 \%)$ & 0 & 0 & 0 \\
TT & $7(6.7 \%)$ & 0 & 0 & 0 \\
BT & $16(14.3 \%)$ & 0 & 1 & 16 \\
BB & $11(10.5 \%)$ & 0 & 7 & 11 \\
BL & $26(25 \%)$ & 20 & 25 & 25 \\
LL & 104 & $20(19 \%)$ & $33(32 \%)$ & $52(50 \%)$ \\
\hline Total & & & 7 & \\
\hline
\end{tabular}

BB: borderline leprosy; BL: borderline lepromatous leprosy; BT: borderline tuberculoid leprosy; LL: lepromatous leprosy; TT: tuberculoid leprosy.

TABLE II

Characteristics of household contacts (HHC) according to test results

\begin{tabular}{|c|c|c|c|c|}
\hline \multirow[b]{2}{*}{ Patient diagnostic } & \multicolumn{4}{|c|}{ Household contacts characteristics } \\
\hline & $\begin{array}{c}\text { Number of HHC } \\
n(\%)\end{array}$ & $\begin{array}{l}\text { IgM anti PGL1 } \\
\text { titers positive (n) }\end{array}$ & Mitsuda negative (n) & $\begin{array}{c}\text { PCR in nasal } \\
\text { swabs positive }(n)\end{array}$ \\
\hline \multicolumn{5}{|l|}{ Ridley Joplin } \\
\hline TT & $177(44 \%)$ & 18 & 19 & 5 \\
\hline BT & $24(6 \%)$ & 4 & 3 & 2 \\
\hline $\mathrm{BB}$ & $45(11 \%)$ & 6 & 3 & 4 \\
\hline BL & $30(7.6 \%)$ & 7 & 3 & 7 \\
\hline LL & $126(31.4 \%)$ & 19 & 10 & 4 \\
\hline Total & 402 & $54(13.4 \%)$ & $38(9.4 \%)$ & $22(5.5 \%)$ \\
\hline
\end{tabular}

BB: borderline leprosy; BL: borderline lepromatous leprosy; BT: borderline tuberculoid leprosy; LL: lepromatous leprosy; TT: tuberculoid leprosy. 
occur in HHC, but individual immune responses are reflected in the seroprevalence and evolution of the infection. This is evident in the variation reported for different geographical populations. The current results show $13.4 \%(n=54)$ of HHC with positive titers for IgM antiPGL1; other studies have shown frequencies of infected contacts ranging from 13 to $93 \%$ (Meeker et al. 1986, Amezcua et al. 1987, Mora et al. 1992, Cardona-Castro et al. 2005). Nevertheless, this number of seropositive HHC does not indicate the true frequency of infected individuals in the studied population since some infected persons do not produce specific antibodies (Oskam et al. 2003). The same situation is true in leprosy patients. Variation in the clinical spectrum of the disease seems to correlate with differences in the immune response, which is affected by the relative contribution of the humoral immune response during disease progression (Oskam et al. 2003).

Twenty-five (46\%) of $54 \mathrm{HHC}$ with positive titers for IgM anti-PGL-I were children under 15 years of age (the youngest was 5 years old). Children represent an especially vulnerable population that require close follow-up to determine if disease has occurred and to begin treatment early in order to avoid the more serious sequelae. However, as mentioned above, IgM-positive titers were not associated with the $\mathrm{BI}$ and clinical diagnosis of the patients, including the population under 15 years of age $(p>0.05)$.

The search for new serological markers of infection is necessary to improve detection of asymptomatic infected contacts. However, the detection of any specific antibodies may be influenced by the same factors that make diagnosis difficult, including variation in the humoral immune response (Oskam et al. 2003). New approaches for the detection of specific antibodies using other antigens and modified methods are under investigation (Parkash et al. 2006, 2007, Geluk et al. 2008).

Acid fast staining of slit skin and nasal swab samples are insufficient for detecting M. leprae in PB patients. As such, it is expected that this test also has low sensitivity for detecting carriers among HHC. None of the HHC studied demonstrated a positive acid fast stain. In contrast, PCR from NS was positive for $22 \mathrm{HHC}$, demonstrating transmission among the population.

With regard to the 22 PCR-positive HHC, 16 (73\%) showed positive titers for anti-PGL1 IgM. This could be due to the presence of $M$. leprae in the nasal passages, resulting in a positive humoral immune response. However, transmission by inhalation has been linked to such cases (Van Beers et al. 1994). Amplification of DNA by PCR also has limitations because it can only detect genetic material, not viable bacteria (Job et al. 1997).

The Mitsuda test is a delayed-type hypersensitivity reaction against intra-dermal injected heat-killed leprosy bacilli, called lepromin. The Mitsuda test induces the formation of T-cell-mediated granulomas (Sengupta 2000). A positive or negative response indicates resistance or susceptibility, respectively, to the lepromatous form of the disease (Feitosa et al. 1996). Therefore, the Mitsuda test can be used for the classification of patients. A Mitsuda reaction in an asymptomatic population such as contacts may be influenced by environmental condi- tions such as exposure to M. leprae. In addition, the immunogenetic background of the individual contributes to the development of a positive skin reaction (Feitosa et al. 1996). Limitations to the interpretation of lepromine in an asymptomatic population can be omitted by using other tests to detect M. leprae infection, such as PCR and anti-PGL1 IgM antibody titers, at the same time. The Mitsuda reaction should be used to determine if a cellular immune response is already present. It is necessary to further develop tests to evaluate the specific cellular immune response in leprosy contacts. Lepromine is an old fashioned test that has risks and ethical concerns associated with its use; however, there are currently no other tests available that demonstrate the cellular immune response in leprosy.

Our study identified three seropositive, Mitsudanegative HHC; these individuals were infected, but demonstrated no cellular immune response to the infection. Such results should stimulate the need for continued follow-up of HHC until clinical signs develop or prophylactic therapy is administrated. In addition, 51 anti-PGL1 IgM-positive HHC demonstrated a positive Mitsuda reaction, showing that they were infected and had an efficient cellular immune response. Follow-up of these $51 \mathrm{HHC}$ is necessary to confirm the development of infection and reduce its transmission.

Several approaches have been used to identify early cases of leprosy, but the available diagnostic tools lack the sensitivity and specificity necessary to achieve this objective. Epidemiological studies have been carried out in endemic regions in order to identify infected contacts; use of ELISA and PCR tests demonstrated the possibility of improved diagnostic accuracy and reduced disease transmission (Chanteau et al. 1993, Van Beers et al. 1994, Wu et al. 1999, Buhrer-Sekula et al. 2000, Beyene et al. 2003, Torres et al. 2003).

Our study suggests that follow-up of high risk populations, such as leprosy contacts, is important, but relying only on clinical examination is not sufficient. Detection of infection and evaluation of the immune response in contacts using several tests at same time is important since the information provided by combined tests provides the opportunity of identifying contacts at a greater risk for infection. Newer tests for infection and the immune response may allow for greater prevention and control of infections. Earlier identification of infection using these combined methods in patients during the course of their disease will lead to better outcomes and reduced transmission.

\section{REFERENCES}

Amezcua ME, Escobar-Gutierrez A, Mayen E, Cazares JV 1987. Sensitivity and specificity of the FLA-ABS test for leprosy in Mexican populations. Int J Lepr Other Mycobact Dis 55: 286-292.

Beyene D, Aseffa A, Harboe M, Kidane D, Macdonald M, Klatser PR, Bjune GA, Smith WC 2003. Nasal carriage of Mycobacterium leprae DNA in healthy individuals in Lega Robi village, Ethiopy. Epidemiol Infect 131: 841-848.

Buhrer-Sekula S, Sarno EN, Oskam L, Koop S, Wichers I, Nery JA, Vieira LM, de Matos HJ, Faber WR, Klatser PR 2000. Use of ML dipstick as a tool to classify leprosy patients. Int J lepr Other Mycobact Dis 68: 456-463. 
Cardona-Castro N, Restrepo-Jaramillo S, Gil de la Ossa M, Brennan P 2005. Infection by Mycobacterium leprae of household contacts of lepromatous leprosy patients from a post-elimination leprosy region of Colombia. Mem Inst Oswaldo Cruz 100: 703-707.

Chanteau S, Glaziou P, Plichart C, Luquiaud P, Plichart R, Faucher JF, Cartel JL 1993. Low predictive value of PGL-I serology for the early diagnosis of leprosy in family contacts: results of a 10-year prospective field study in French Polynesia. Int J Lepr Other Mycobact Dis 61: 533-541.

Cho SN, Yanagihara DL, Hunter SW, Gelber RH, Brennan PJ 1983. Serological specificity of phenolic glycolipid I from Mycobacterium leprae and use in serodiagnosis of leprosy. Infect Immun 41:1077-1083.

Feitosa M, Krieger H, Borecki I, Beiguelman B, Rao DC 1996. Genetic epidemiology of the Mitsuda reaction in leprosy. Hum Hered 46: 32-35.

Geluk A, van der Ploeg J, Teles RO, Franken KL, Prins C, Drijfhout JW, Sarno EN, Sampaio EP, Ottenhoff TH 2008. Rational combination of peptides derived from different $M$. leprae proteins improves sensitivity for immunodiagnosis of $M$. leprae infection. Clin Vaccine Immunol 15: 522-533.

Groathouse NA, Rivoire B, Hansuk K, Hyeyoung L, Cho SN, Brennan PJ, Vissa VD 2004. Multiple polymorphic loci for molecular typing of strains of Mycobacterium leprae. J Clin Microbiol 42: 1666-1672.

Gupta V, Bhushan K, Dogra S, Vasudev U, Kaur I 2004. Leprosy elimination - How far? Lep Rev 75: 101-102.

Jadhav RS, Macdonald M, Bjune G, Oskam L, MILEP2 Study Group 2001. Simplified PCR detection method from nasal Mycobacterium leprae. Int J Lepr Other Mycobact Dis 69: 299-307.

Job CK, Jayakumar J, Williams DL, Gillis TP 1997. Role of polymerase chain reaction in the diagnosis of early leprosy. Int J Lepr 65: 461-464.

Kalk A, Fleisher K 2004. The decentralization of the health system in Colombia and Brazil and its impact on leprosy control. Lepr Rev 75: 67-68.

Lockwood DN 2002. Leprosy elimination - a virtual phenomenon or a reality? BMJ 324: 1516-1518.

Lockwood DNJ, Kumar B 2004. Treatment of leprosy. BMJ 328: 1447-1448.

Meeker HC, Levis WR, Sersen E, Schuller-Levis G, Brennan PJ, Buchanan TM 1986. ELISA detection of IgM antibodies against phe- nolic glycolipid-I in the management of leprosy: a comparison between laboratories. Int J Lepr Other Mycobact Dis 54: 530-539.

Meima A, Smith WCS, van Ootmarssen GJ, Richardus JH, Habbema DF 2004. The future incidence of leprosy: a scenario analysis. Bull World Health Organ 5: 373-380.

Mitsuda K 1953. On the value of a skin reaction to a suspension of leprous nodules. Int J Lepr 21: 347-350.

Mora N, Pérez M, Beroliche M 1992. Determinación de anticuerpos anti-glicolípido fenólico I en población general de una área endémica de lepra. Fontilles Rev Leprol 6: 587-589.

Naafs B 2000. Viewpoint: leprosy after the year. Trop Med Int Health 5: $400-403$.

Oskam L, Slim E, Bührer-Sékula S 2003. Serology: recent developments, strengths, limitations and prospects: a state of the art overview. Lepr Rev 74: 196-205.

Parkash O, Kumar A, Nigam A, Franken KL, Ottenhoff TH 2006. Evaluation of recombinant serine-rich 45-kDa antigen (ML0411) for detection of antibodies in leprosy patients. Scand J Immunol 64: $450-455$.

Parkash O, Kumar A, Pandey R, Franken KL, Ottenhoff TH 2007. Detection of Mycobacterium leprae infection employing a combinatorial approach of anti-45 kDa and modified anti-PGL-I antibody detection assays. J Med Microbiol 56: 1129-1130.

Scollard DM, Gillis TP, Williams DL 1998. Polymerase chain reaction assay for the detection and identification of Mycobacterium leprae in patients in the United States. Am J Clin Pathol 109: 642-646.

Sengupta U 2000. Experience and lessons from the use of lepromin and Mycobacterium leprae-specific serology. Lepr Rev 71: S63-66.

Torres P, Camarena JJ, Gomez JR, Nogueira JM, Gimeno V, Navarro V, Navarro JC, Olmos A 2003. Comparison of PCR mediated amplification of DNA and the classical methods for detection of Mycobacterium leprae in different types of clinical samples in leprosy patients and contacts. Lepr Rev 74: 18-30.

Van Beers SM, Izumi S, Madjid B, Maeda Y, Day R, Klatser PR 1994. An epidemiological study of leprosy infection by serology and polymerase chain reaction. Int J Lepr Other Mycobact Dis 62: 1-9.

Visschedijk J, van de Broek, Eggens H, Lever P, van Beers S, Klatser P 2000. Mycobacterium leprae-millennium resistant! Leprosy control on the threshold of a new era. Trop Med Int Health 5: 388-399.

Wu Q, Li X, Yin Y, Shu H, Wei W, Liu Q, Ye G 1999. A study on the methods for early serological diagnosis of leprosy and their potential use. Int J Lepr Other Mycobact Dis 67: 302-305. 